\title{
Prevalence of dry eye syndrome in type-2 diabetic patients in ter- tiary care hospital kurnool
}

\section{Pushpa Latha $\mathrm{M}^{1}$, Radhika $\mathrm{R}^{* 2}$.}

${ }^{1}$ Assistant Professor, Department of Ophthalmology, Viswabharathi Medical college, Kurnool, Andhra Pradesh, India.

*2 Senior consultant, Kranti Eye Clinic, Kurnool, Andhra Pradesh, India.

\section{ABSTRACT}

Introduction: The examination of dry eyes should be an integral part of the assessment of diabetic eye disease. So, this study is undertaken to study the prevalence of dry eye syndrome in type II diabetes mellitus.

Materials and Methods: This hospital based Cross-sectional descriptive study conducted with 100 patients with type II diabetes and 100 matched controls patients who were attending the Ophthalmology Department in Viswabharthi medical college Kurnool by Simple random sampling method. The data collected and analyzed using SPSS 17.0 version software for analysis.

Results: The mean age of diabetics with dry eye was $56.49 \pm 9.32$ years. As the age increased, the occurrence of dry eye also increased significantly. 30-40yrs age group are having 2 (33.33\%) patients, $41-50$ yrs age group are having $16(59.26 \%)$ patients, 51-60 yrs age group are having $19(57.58 \%)$ and above 60 years age group are having 20 (58.83\%) patients are having dry eye, out of this $64.7 \%$ also had retinopathy of some degree. With increasing age, the number of patients with diabetic retinopathy also increased significantly.

Conclusion: The dry eye is a significant factor responsible for ocular surface disease in diabetics, these complications high in advanced diabetic retinopathy. High prevalence could be attributed to reduced tear secretion in DM patients caused by autonomic dysfunction in these patients. Hence our study insists that clinical evaluation of dry eye should be an integral part of ocular examination in diabetic patients.

KEY WORDS: Dry eye, ocular surface disease index, Retinopathy, Schirmer, Tearfilm breakup time.

Address for correspondence: Dr. Radhika R, Senior consultant, Kranti Eye Clinic, H. No: 4-23B-22, Maruthinagar, Near Balajinagar, Kurnool-518006, Andhra Pradesh, India.

E-Mail: pushpa0502@gmail.com

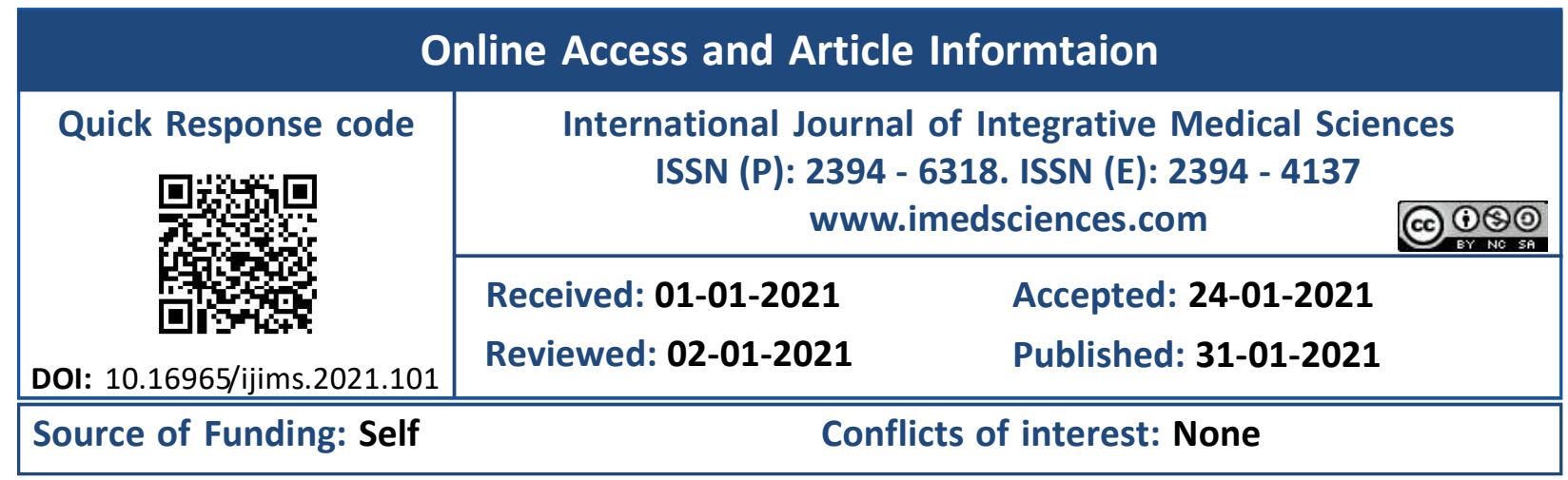

\section{INTRODUCTION}

Cataract and retinopathy are well known as ocular surface complications of diabetes mellitus (DM) is a common microangiopathy which not only involves the inner structure of the eye but also the cornea and other extra ocular adnexae. Recently problems involving the ocular surface, dry eyes disease (DED) in particular have been reported in DM patients. These patients are also having increased risk of developing corneal epithelial fragility, decreased corneal sensitivity, abnormal wound healing, and increased susceptibility to infected corneal ulceration [1]. Reduced goblet cell density in the conjunctiva along with meibomiam gland dysfunction accounts for the reduced tear break-up time seen in these individuals. It is also proposed that longstanding disease may cause damage to the microvascular supply to the lacrimal gland, impairing lacrimation [1].

$\mathrm{DM}$ is one of the most common leading causes 
of blindness in 20-74 years old persons and around $47 \%-64 \%$ of patients with DM had keratopathies in their lifetime [2]. More than $50 \%$ of diabetic patients are also diagnosed with DED, which shows a significant correlation with the duration of diabetes. Dry eye is an important contributor to these problems. Structural, metabolic and functional abnormalities are found in the conjunctiva and cornea of diabetic patients. DED is a characterized by dysfunction in tear production or tear film instability [3]. Elevated levels of inflammatory cytokines such as interleukin (IL)-1, IL-6, IL-8, IL-10, interferon- $\alpha, \gamma$, and tumor necrosis factor (TNF)- $\alpha$ are found in tears of patients with DED due to Low tear production or excessive evaporation increases tear osmolarity. So, the examination of dry eyes should be an integral part of the assessment of diabetic eye disease. So, this study is undertaken to study the prevalence of dry eye syndrome in type II diabetes mellitus.

\section{METHOD OF COLLECTION OF DATA}

This is a hospital based Cross-sectional descriptive study which included 100 patients with type II diabetes and 100 matched controls patients who were attending the Ophthalmology Department in Viswabharthi medical college Kurnool with were included in the study in one period (June 2019 to June 2020). After clearance from the Institutional Ethical Committee in accordance with the guidelines of the Declaration of Helsinki and taking informed consent, all type 2 diabetic patients irrespective of age, sex, duration of diabetes were included in the study. Type 1 diabetes, Secondary diabetes, associated with other diseases- sjogrens syndrome, rheumatoid arthritis, lupus, Parkinson's, on medications antihistaminics, tricyclic antidepressants, ocps, Steroids, ACTH, Pregnancy, and Vitamin A deficiency persons were not included. Detailed history of each patient was obtained regarding the age, sex, occupation and presenting symptoms, duration, progression and associated conditions. Detailed history regarding diabetes such as type, duration, type of treatment, $\mathrm{HbA1c}$ values, FBS and PPBS levels were recorded.
Ocular surface disease index (OSDI) questionnaire was used to score the dry eye symptoms. OSDI was calculated using the formula OSDI = (total score) $\times 25$ the whole divided by the number of questions answered. Using the OSDI score the patients were categorized as normal (0-12), mild dry eye (13-22), moderate dry eye (23-32) and severe dry eye (33-100). The dry eye was detected by measuring tear film breakup time (TBUT), ocular surface dye staining pattern with fluorescein and Schirmer's test. A value $<10$ seconds was $c$ onsidered abnormal. TBUT results graded as $>10$ secs- normal, 6-10 secs - mild to moderate, and $<6$ secs as severe. Ocular surface staining was evaluated by staining the cornea with fluorescein. The staining area was graded using the Oxford Scheme on a numerical scale of $0-5$ for the entire ocular surface based on comparison to the standard panel, with 0 representing equal to or less than panel $A, 1$ representing equal to or less than panel $B$ but greater than panel $A, 2$ representing equal to or less than panel C but greater than panel B, 3 representing equal to or less than panel $D$ but greater than panel $D, 4$ representing equal to less than panel $\mathrm{E}$ but greater than panel $\mathrm{C}$, 5 representing greater than panel $\mathrm{E}$. The severity was graded based on the score as 0-1 as normal, 2 as mild, 3- moderate and 4 as severe.

Schirmer test was performed without topical anesthesia using standardized Whatman filter paper 41 . The strips were placed in the lower fornix away from the cornea and left in place for 5 min with the patient opened eyes. The wetting distance was measured in millimeters, and a reading $<10 \mathrm{~mm}$ was considered abnormal.

Statistical method used: Simple random sampling method. The data collected and analyzed using SPSS (Statistical Package for Social Sciences) $\mathbf{1 7 . 0}$ version software for analysis. The means and proportions were calculated. The test of significance used has chi- square test. A p value of less than 0.05 will be considered significant.

\section{RESULTS}

A total of 100 patients with diabetes were 
included in the study, 57 (57.0\%) patients were diagnosed to have dry eye. Out of 100 diabetic patients, 53 diabetic patients with retinopathy, 41 patients $(77.4 \%)$ had dry eye. 47 diabetic patients without retinopathy, 16 patients (34\%) were found to have dry eye. Out of 100 consecutive patients included in the study, 55 were male and 45 were female. Among 55 male patients, 26 patients (52.7\%) had diabetic retinopathy and 31 patients (56.37\%) had dry eye and among 45 females' patients, 24 patients (53.3\%) had diabetic retinopathy and 26 patients (57.78\%) had dry eye symptoms.

The age group of these 100 diabetic patients is above 30 years. The mean age of diabetics without dry eye was $54.77 \pm 9.82$ years and the mean age of diabetics with dry eye was $56.49 \pm 9.32$ years. As the age increased, the occurrence of dry eye also increased significantly. Out of 100 patients 6 were $30-40 y r s$ age group are having 2 (33.33\%) patients, 27 were 41-50 yrs age group are having 16 (59.26\%) patients, 33 were 51-60 yrs age group are having 19 (57.58\%) and 34 were above 60 years age group are having 20 (58.83\%) patients are having dry eye. Mean age in diabetic patients without retinopathy was $53.72 \pm 9.88$ years,

Table 1: Dry eye among the Diabetics on ocular surface disease index (OSDI).

\begin{tabular}{ccccc}
\hline S. No & OSDI Grading & $\begin{array}{c}\text { Number of } \\
\text { patients }\end{array}$ & $\begin{array}{c}\text { \% of } \\
\text { patients }\end{array}$ & Total \% \\
\hline $\mathbf{1}$ & Normal & 49 & $49.00 \%$ & $49.00 \%$ \\
$\mathbf{2}$ & Mild & 20 & $20.00 \%$ & \\
$\mathbf{3}$ & Moderate & 20 & $20.00 \%$ & $51.00 \%$ \\
$\mathbf{4}$ & Severe & 11 & $11.00 \%$ & \\
\hline
\end{tabular}

Table 2: Dry eye among the Diabetics using Tearfilm breakup time (TBUT).

\begin{tabular}{ccccc}
\hline S. No & TBUT Grading & $\begin{array}{c}\text { Number of } \\
\text { patients }\end{array}$ & $\begin{array}{c}\text { \% of } \\
\text { patients }\end{array}$ & Total \% \\
\hline $\mathbf{1}$ & Normal & 58 & $49.00 \%$ & $49.00 \%$ \\
$\mathbf{2}$ & Mild -Moderate & 38 & $38.00 \%$ & $42.00 \%$ \\
\hline $\mathbf{4}$ & Severe & 4 & $4.00 \%$ & \\
\hline
\end{tabular}

while in diabetic patients with retinopathy was $57.55 \pm 8.93$ years. With increasing age, the number of patients with diabetic retinopathy also increased significantly. Out of 34 patients in the age group more than 60 years, 24 patients $(64.7 \%)$ had retinopathy of some degree.

\section{DISCUSSION}

Previously, various studies have reported increased incidence of dry eye syndrome in diabetic patients. In the present study, the prevalence of dry eye in diabetics was $57 \%$. In particular, diabetics with retinopathy, had higher prevalence (77.4\%) of dry eye than diabetics without retinopathy. A study by Seifart et al showed that $52.8 \%$ of all diabetic subjects complained of dry eye symptoms, as against $9.3 \%$ of the controls and concluded that close monitoring of diabetic patients and good blood sugar regulation is important for the prevention of dry eye syndrome and retinopathy [4] and Khurana $G$ et al. [5] also found a higher prevalence of dry eye disorder in diabetic patients. High prevalence of dry eye disorder can be explained by low tear production DM patients related to dysfunction of the autonomic nervous system. The tropical and dry climate of our region could be the reason for high prevalence of dry eye seen in present study.

Based on the OSDI symptom scores, among the 100 diabetic patients $51 \%$ of patients had symptomatic dry eye. Among them, mild dry eye was noted in $20 \%$ of patients, moderate dry eye in $20 \%$ of patients and $11 \%$ had severe dry eye. The frequency of dry eye symptoms in this study matched to that of Manaviat MR et al, who found that $54 \%$ of 199 diabetic subjects had dry eye symptoms [6]. This study also showed significant difference in the OSDI

Table 3: Showing Clinical Characteristics of Study Population.

\begin{tabular}{|c|c|c|c|c|c|c|}
\hline & \multicolumn{2}{|c|}{$\begin{array}{l}\text { Control group } \\
\qquad(n=100)\end{array}$} & \multicolumn{2}{|c|}{$\begin{array}{l}\text { Diabetic without } \\
\text { retinopathy }(n=47)\end{array}$} & \multicolumn{2}{|c|}{$\begin{array}{l}\text { Diabetic with } \\
\text { retinopathy }(n=53)\end{array}$} \\
\hline & Mean & SD & Mean & SD & Mean & SD \\
\hline Age (yrs) & 54.7 & 10.23 & 53.72 & 9.88 & 57.55 & 8.93 \\
\hline Duration of Diabetes (yrs) & - & - & 4.86 & 5.23 & 8.79 & 5.53 \\
\hline HbA1C (\%) & - & - & 8.85 & 2.41 & 9.2 & 2.27 \\
\hline Schirmer (mm) & 24.15 & 5.15 & 11.67 & 4.41 & 10.04 & 3.42 \\
\hline TBUT (sec) & 14.65 & 3.68 & 13.94 & 4.66 & 11.44 & 4.23 \\
\hline OSDI & 6.98 & 6.52 & 8.04 & 8.26 & 21.37 & 12.16 \\
\hline
\end{tabular}


scores between the diabetics with retinopathy and the non-diabetic group while no significant difference between the diabetics without retinopathy and the controls. Jin et al reported that TBUT is significantly lower in type 2 diabetic patients as compared to controls [7] while Goebels reported that Schirmer test and tearing reflex was significantly lower in diabetic patients compared with control group [8] and Khurana $\mathrm{G}$ et al [5] also found abnormal Schirmer's test in $26.25 \%$ of patients with abnormal TBUT in $62.50 \%$ patients. We found normal TBUT value $(<10 \mathrm{sec})$ was seen in $49 \%$ of the diabetic group. Schirmer test was abnormal $(<100 \mathrm{~mm} / 5 \mathrm{~min})$ in $54 \%$ of diabetic subjects. Dogru et al also reported that TBUT and Schirmer test values are significantly lower in the diabetic patients, especially in patients with peripheral neuropathy and poor metabolic control [9]. Manaviat MR et al [6] concluded that $59.3 \%$ of patients with diabetic retinopathy suffered from dry eye syndrome which was higher than patients without diabetic retinopathy.

Deterioration of tear film status was found to be significantly associated with severity of diabetic retinopathy by Ozdemir et al [10] and Khurana $G$ et al [5] study found similar results showing that the patients with diabetic retinopathy had a 6.65 times more chance of having Schirmer's BST value $=10 \mathrm{~mm}$ than those without diabetic retinopathy. Also, patients with diabetic retinopathy had 6.37 times more chance of having TBUT $=10$ seconds than those without diabetic retinopathy. Presence of autonomic dysfunction, reduced corneal sensitivity, abnormalities of tear film dynamics, damage to the microvasculature of the lacrimal gland and subclinical meibomian gland dysfunction can all attribute to increased prevalence and severity of dry eye disorders in patients with diabetes.

\section{CONCLUSION}

Present study is showing that abnormal Schirmer test, OSDI scores, and TBUT were noticed in diabetic patients compared to controls. These observations indicate that dry eye is a significant factor responsible for ocular surface disease in diabetics. High prevalence could be attributed to reduced tear secretion in DM patients caused by autonomic dysfunction in these patients. Patients with advanced diabetic retinopathy are at increased risk of developing ocular surface complications. Hence our study insists that clinical evaluation of dry eye should be an integral part of ocular examination in diabetic patients

Limitations of the study: Cross sectional design of the study, Limited sample size, Lack of more objective tests like conjunctival impression cytology and tear osmolarity measurement are limitations of our study.

\section{REFERENCES}

[1]. Manchikanti V, Kasturi N, Rajappa M, Gochhait D. Ocular surface disorder among adult patients with type II diabetes mellitus and its correlation with tear film markers: A pilot study. Taiwan J Ophthalmol.2020.

[2]. Schultz RO, Peters MA, Sobocinski K, Nassif K, Schultz KJ. Diabetic corneal neuropathy. Trans Am Ophthalmol Soc 1983;81:107-24.

[3]. Jae Hoon Han, Yong Chan Cho, Won-Gun Koh, Young Bin Choy.Preocular sensor system for concurrent monitoring of glucose levels and dry eye syndrome using tear fluids. PLoS One. 2020; 15(10): e0239317.

[4]. Seifart U, Strempel I: The dry eye syndrome and diabetes mellitus. Ophthalmologe 1994; 91:235-9.

[5]. Geetika Khurana, Deeksha Khurana, Rajesh Jain Dry Eye in Patients with Diabetic Retinopathy: A Clinical Study. DJO 2017;27(3):190-3.

[6]. Manaviat MR, Rashidi M, Afkhami-Ardekani M, Shoja MR. Prevalence of DE syndrome and diabetic retinopathy in type 2 diabetic patients. BMC Ophthalmol. 2008; 8: 10.

[7]. Jin J, Chen LH, Liu XL, Jin GS, Lou SX, Fang FN: Tear film function in non-insulin dependent diabetics. Zhonghua Yan Ke Za Zhi 2003; 39:10-3.

[8]. Goebbels M: Tear secretion and tear film function in insulin dependent diabetics. Br J Ophtalmol 2000; 84:19-21.

[9]. Dogru M, Katakami C, Inoue M. Tear function and ocular surface changes in noninsulin-dependent diabetes mellitus. Ophthalmology 2001; 108:58692.

[10]. Ozdemir M, Buyukbese MA, Cetinkaya A, Ozdemir G. Risk factors for ocular surface disorders in patients with diabetes mellitus. Diabetes Res Clin Pract 2003; 59:195-9.

How to cite this article: Pushpa Latha M, Radhika R. Prevalence of dry eye syndrome in type-2 diabetic patients in tertiary care hospital kurnool. Int J Intg Med Sci 2021;8(1):955-958. DOI: 10.16965/ijims.2021.101 